\title{
Cardiovascular efficacy of sitagliptin in patients with diabetes at high risk of cardiovascular disease: a 12-month follow-up
}

Takashi Nakamura $^{1 \dagger}$, Yoshitaka Iwanaga ${ }^{1 \dagger}$, Yuki Miyaji ${ }^{1}$, Ryuji Nohara ${ }^{2}$, Takao Ishimura ${ }^{3}$, Shunichi Miyazaki ${ }^{1 *}$ and on behalf of the Sitagliptin Registry Kinki Cardiologists'Study (SIRKAS) Investigators

\begin{abstract}
Background: Gliptins should have beneficial effects beyond glycemic control, potentially on the pathophysiology of cardiovascular (CV) diseases, with some basic studies demonstrating this possibility. However, we are yet to answer whether there are any direct CV effects in the clinical setting. We aimed to examine the beneficial effects of sitagliptin in Japanese patients with diabetes and high CV risk for 12 months.
\end{abstract}

Methods: This was a prospective, multicenter, observational study of 205 patients with type 2 diabetes. All participants had more than one major CV risk factor and were treated with sitagliptin for 12 months. At 3 or 12 months, we examined the effects of treatment on glycemic control, CV function (by electrocardiography, echocardiography, and reactive hyperemia-peripheral arterial tonometry), and CV biomarkers.

Results: Patients were predominantly elderly (68.8 \pm 9.9 years) and male $(71.5 \%)$ and typically had more than three CV risk factors (79.2\%). Treatment with sitagliptin significantly reduced the hemoglobin A1c (HbA1c) level from $7.09 \% \pm 0.81 \%$ at baseline to $6.67 \% \pm 0.69 \%$ at 3 months and $6.68 \% \pm 0.73 \%$ at 12 months (both $P<0.001$ ). The reduction in $\mathrm{HbA} 1 \mathrm{c}$ was also in tandem with the decrease in the level of high-sensitive $\mathrm{C}$-reactive protein throughout the study. Independent of the change in $\mathrm{HbA1c}$, sitagliptin reduced systolic $(-7.0 \pm 18.9 \mathrm{mmHg})$ and diastolic blood pressure $(-5.1 \pm 11.7 \mathrm{mmHg})$ at 12 months, and this was associated with a decrease in urinary albumin. In contrast, there were no beneficial effects on cardiac and endothelial function or on the levels of serum B-type natriuretic peptide, high-sensitive troponin T, and urinary 8-hydroxy-2'-deoxyguanosine.

Conclusions: In Japanese patients with diabetes and multiple CV risk factors, sitagliptin showed a decrease in blood pressure associated with an improvement in albuminuria in addition to glycemic control.

Trial registration: UMIN000005663

Keywords: Blood pressure, Cardiovascular risk, Type 2 diabetes, Sitagliptin, Urine albumin

\section{Background}

Type 2 diabetes is strongly associated with coronary atherosclerosis and vascular complications, both of which are responsible for worse morbidity and mortality [1].

\footnotetext{
*Correspondence: smiyazak@med.kindai.ac.jp

†Takashi Nakamura and Yoshitaka Iwanaga contributed equally to this work

1 Division of Cardiology, Department of Internal Medicine, Faculty of Medicine, Kinki University, 377-2 Ohno-Higashi,

Osakasayama 589-8511, Japan

Full list of author information is available at the end of the article
}

Although the pathogenesis of atherosclerosis and associated complications remains unclear [2], inflammatory processes may be a critical step, constituting a biological link between diabetes and vascular disease [3]. Optimal management of type 2 diabetes includes pharmacological interventions and life-style modification, including diet, exercise, and weight loss. To reduce the risk of cardiovascular (CV) events, not only is pharmacological therapy necessary for lowering blood sugar levels but antihypertensive, antihyperlipidemic, and antiplatelet therapies are 
also needed [4]. In particular, integrated behavior modification and targeted polypharmacy are especially beneficial for patients with diabetes who are at high risk of $\mathrm{CV}$ disease (CVD) [5].

Sitagliptin, a dipeptidyl peptidase-4 (DPP-4) inhibitor (gliptin), has been shown to increase plasma incretin hormone levels, including glucagon-like peptide-1 (GLP-1) and glucose-dependent insulinotropic polypeptide (GIP). Increases in these peptides appear to be responsible for the principle mechanism of action of sitagliptin; as the levels of GLP-1 increase, glucose concentrations decrease [6]. Sitagliptin is known to be effective and safe as both monotherapy and combination therapy in patients with diabetes. Recently, the Trial Evaluating Cardiovascular Outcomes with Sitagliptin (TECOS) assessed composite $\mathrm{CV}$ endpoint and hospitalization rates for heart failure in patients with type 2 diabetes and established CVD, and demonstrated that sitagliptin was safe [7]. However, we still do not know the various long-term influences of incretin-based therapies, such as sitagliptin, on CV risk factors and disease, including the effects on morbidity and mortality.

DPP-4 is a widely expressed glycoprotein that exists either as a transmembrane protein or in a soluble form in plasma, and has many targets other than GLP-1 and GIP. In addition, the GLP-1 receptor is variously expressed, being located not only in pancreatic cells but also in the lungs, kidneys, intestines, peripheral and central nervous systems, and the CV system. Based on this, it has been suggested that gliptins should have beneficial effects beyond glycemic control, potentially on the pathophysiology of CVD, with some basic studies demonstrating this possibility [8]. However, these effects remain unclear in the clinical setting [9], and the factors predictive of a blood glucose-lowering effect remain unknown in patients with diabetes who are at high risk of CVD. Equally, we are yet to answer whether there are any direct $\mathrm{CV}$ effects in the medium term.

We performed this prospective study to determine whether sitagliptin therapy for 12 months had any beneficial effects on glycemic control and CV function in Japanese patients with diabetes at high risk of CVD.

\section{Methods}

\section{Study design}

This was a prospective, multicenter, observational study. Eligible patients with diabetes at high risk of CVD were treated with sitagliptin for 12 months from August 2011 to September 2012, at nine hospitals and clinics in Japan. The patients received sitagliptin at dosages of 25,50 , or $100 \mathrm{mg} /$ day at the discretion of the attending physician, and biochemical markers, urinalysis, electrocardiography (ECG), and echocardiography (UCG) were assessed before, and at 3 and 12 months after treatment. In a substudy at the Department of Internal Medicine, Kinki University Faculty of Medicine and the Tazuke Kofukai Medical Research Institute, Kitano Hospital, we also performed reactive hyperemia-peripheral arterial tonometry (RH-PAT) before and at 3 months after starting sitagliptin and measured CV biomarkers before and at 3 and 12 months after treatment.

The study protocol was approved by the Ethics Committee of Kinki University Faculty of Medicine and Osaka Foundation for the Prevention of Cancer and Cardiovascular Disease. Written informed consent was obtained from all patients. The trial was registered at http://www. umin.ac.jp under UMIN000005663.

\section{Selection criteria}

The inclusion criteria were as follows: (1) age $\geq 20$ years; (2) type 2 diabetes with a hemoglobin A1c (HbA1c) $\geq 6.2 \%$, a fasting glucose $\geq 110 \mathrm{mg} / \mathrm{dL}$, or a postprandial glucose $\geq 140 \mathrm{mg} / \mathrm{dL}$ despite appropriate diet and exercise, oral antihyperglycemic agents, or insulin therapy; and (3) at least one CV risk from among hypertension, dyslipidemia, previous $\mathrm{CV}$ event, advanced age ( $\geq 65$ years), current smoking, obesity (body mass index $\geq 25$ ), and hyperuricemia. Patients were excluded if they met the following criteria: (1) history of diabetic coma and ketoacidosis within 6 months before the study, (2) serious trauma or infectious disease, (3) currently nursing or pregnant women, (4) dialysis or severe renal insufficiency (creatinine clearance $<30 \mathrm{~mL} / \mathrm{min}$ ), and (5) known hypersensitivity to any component of the study medications.

\section{Study endpoints}

The primary endpoint was the change in HbA1c after 3 and 12 months of sitagliptin therapy. The major secondary endpoints were changes in body weight, blood pressure (BP), biochemical measures, urinary measures, ECG parameters, and UCG parameters.

\section{Ambulatory BP measurement}

BP was measured during office visits at baseline and after 3 and 12 months of treatment. Cuffs were used with the bladder encircling at least $80 \%$ of the mid-arm circumference and placed directly on the skin. All BP measurements were taken after $5 \mathrm{~min}$ of rest with patients sitting and with the arm resting on a table at the level of the heart.

\section{Measurement of biochemical and urine parameters}

The following parameters were measured at baseline and after 3 and 12 months of treatment: standard urinalysis, urinary albumin, complete blood count, serum electrolytes, liver function, renal function, lipid 
profile, immunoreactive insulin, and HbAlc. In the sub-study, we also measured the following before and at 3 and 12 months after treatment: urinary 8-hydroxy2 '-deoxyguanosine $(8-\mathrm{OHdG})$ as a marker of oxidative stress, high-sensitive C-reactive protein (hs-CRP) as an inflammatory marker, and both high-sensitive troponin $\mathrm{T}$ (hs-TnT) and B-type natriuretic peptide (BNP) as cardiac markers. The estimated glomerular filtration rate (eGFR) was calculated using the equation specific to the Japanese population: eGFR $=194 \times($ serum creatinine $)^{-1.094} \times(\text { age })^{-0.287}(\times 0.739$ if female $)[10]$. Albuminuria was determined by the urinary albuminto-creatinine ratio (UACR). Microalbuminuria was defined as a UACR of $30-300 \mathrm{mg} / \mathrm{g}$ creatinine, and overt albuminuria as a UACR of $300-3000 \mathrm{mg} / \mathrm{g}$ creatinine. The homeostasis model assessment-insulin resistance (HOMA-IR) value was calculated using the following formula: HOMA-IR $=$ [fasting insulin $(\mu \mathrm{IU} / \mathrm{ml}) \times$ fasting glucose $(\mathrm{mg} / \mathrm{dl})] / 405$.

\section{ECG and UCG examination}

ECG and UCG were performed at baseline and after 12 months of treatment. The ECG parameters were examined by automatic analysis [ECG-1450 (Nihon Kohden, Tokyo, Japan)]. Heart rate (HR), rhythm, PR interval, QRS interval, QT/corrected QT (QTc) interval, and the SV1 + RV5 value were obtained [11]. In the UCG analysis, the chamber dimensions, left ventricular (LV) ejection fraction (EF), and LV mass index were assessed from M-mode images using the American Society of Echocardiography-recommended formula [12]. For patients in sinus rhythm, the pulsed Doppler transmitral flow velocity was recorded to measure the ratio of peak mitral E-wave velocity to peak mitral A-wave velocity (E/A ratio) and the deceleration time of the mitral E-wave velocity (Dct). The peak of myocardial early diastolic velocity $\left(\mathrm{e}^{\prime}\right)$ in the septal annulus was also measured by tissue Doppler imaging, and the ratio of peak mitral E-wave velocity to $\mathrm{e}^{\prime}$ velocity (E/e' ratio) was calculated for the assessment of $\mathrm{LV}$ relaxation.

\section{Assessment of endothelial function}

In the sub-study, we assessed peripheral endothelial function by RH-PAT using an EndoPAT2000 (Itamar Medical, Caesarea, Israel), as described previously [13]. RH-PAT was performed at baseline and after 3 months of treatment to calculate the reactive hyperemia index (RHI), which reflects the extent of reactive hyperemia, and thereby, endothelial function. It was calculated as the ratio of the average amplitude of the PAT signal over 1 min starting at 1.5 min after cuff deflation (control arm, A; occluded arm, C) divided by the average amplitude of the PAT signal of a 2.5-min time period before cuff inflation (baseline) (control arm, B; occluded arm, D), as follows: $\mathrm{RHI}=(\mathrm{C} / \mathrm{D}) /(\mathrm{A} / \mathrm{B})$.

\section{Statistical analysis}

Categorical variables were compared using the $\chi^{2}$ test for proportions and the unpaired $t$ test or analysis of variance (ANOVA) for continuous variables, as appropriate. The linearity of the relationship between two variables was assessed by linear regression analysis, and Pearson's correlation coefficient was calculated. Differences between the reduction or increase in each marker and the change in HbA1c were compared using Student's $t$ tests. Further multiple logistic regression analysis was performed to define independent variables that might predict the change in HbA1c. Overall differences between before and after sitagliptin administration were determined using repeated measures ANOVA. All P values $<0.05$ were considered statistically significant. Results are expressed as the mean \pm standard deviation (SD). All analyses were performed using JMP version 10.0.

\section{Results}

\section{Baseline clinical characteristics}

Of the 211 patients enrolled, 6 patients were excluded from the analysis because they discontinued sitagliptin after 3 months, citing dizziness ( 2 patients), mild hypoglycemia (1 patient), headache (1 patient), or general malaise (1 patient). However, no severe adverse events, including severe hypoglycemia, were reported. One patient withdrew consent. The baseline characteristics of the 205 participants are shown in Table 1. Most were male $(71.5 \%)$ and aged over 65 years $(73.4 \%)$, with mean systolic and diastolic BPs of $133.6 \pm 19.2$ and $74.8 \pm 11.6 \mathrm{mmHg}$, respectively. Three or more CV risk factors were present in $79.2 \%$, with hypertension being most common. However, $42.0 \%$ had some medical history of CVD, including coronary heart disease. Over the study period, the mean sitagliptin dosage was $52.0 \pm 12.7 \mathrm{mg} /$ day. About half of the patients (52.6 \%) received sitagliptin monotherapy and the remainder received combination therapy, with the most common co-administered drugs being sulfonylureas (21.3\%) followed by $\alpha$-glucosidase inhibitors $(17.9 \%)$ and thiazolidinediones (14.5\%).

\section{Blood glucose and urinary changes (Table 2)}

At baseline, HbA1c and fasting blood glucose were $7.09 \pm 0.81 \%$ and $139.8 \pm 33.0 \mathrm{mg} / \mathrm{dL}$, respectively, and both parameters significantly reduced by 3 and 12 months (both $\mathrm{P}<0.001$ ). However, there were no changes in insulin, HOMA-IR, low-density lipoprotein cholesterol, triglyceride, or the uric acid level. Only high-density lipoprotein (HDL) cholesterol level reduced 
Table 1 Baseline clinical characteristics

\begin{tabular}{|c|c|c|}
\hline & $N=205$ & \\
\hline Age (years) & $68.8 \pm 9.9(37-88)$ & \\
\hline Male (\%) & 71.5 & \\
\hline BMI $\left(\mathrm{kg} / \mathrm{m}^{2}\right)$ & $25.7 \pm 4.1$ & \\
\hline Blood pressure (systolic/diastolic) (mmHg) & $133.6 \pm 19.2 / 74.8 \pm 11.6$ & \\
\hline Cardiovascular risk factors $\geq 3(\%)$ & 79.2 & \\
\hline Hypertension & 87.0 & \\
\hline Dyslipidemia & 65.2 & \\
\hline Cardiovascular disease & 42.0 & \\
\hline Coronary artery disease & 39.6 & \\
\hline ASO & 2.4 & \\
\hline Cerebral infarction & 2.9 & \\
\hline Senior ( $\geq 65$ years) & 73.4 & \\
\hline Current smoking & 16.6 & \\
\hline Obesity (BMI $\geq 25)$ & 52.9 & \\
\hline Hyperuricemia ( $\geq 7.0$ mg/dL) & 19.9 & \\
\hline Combination of oral hypoglycemic drugs (\%) & Pre- & Post- \\
\hline None & 52.6 & 56.3 \\
\hline Sulfonylurea & 21.3 & 19.4 \\
\hline a-Glucosidase inhibitor & 17.9 & 16.5 \\
\hline Thiazolidinedione & 14.5 & 12.6 \\
\hline Biguanide & 10.6 & 10.6 \\
\hline Glinide & 6.3 & 2.4 \\
\hline ACEI/ARB & 63.6 & \\
\hline Calcium channel blocker & 41.5 & \\
\hline Statin & 58.3 & \\
\hline
\end{tabular}

Values are the mean \pm SD or $\%$

$A C E I$ angiotensin-converting enzyme inhibitor, $A R B$ angiotensin receptor blocker, $A S O$ arteriosclerosis obliterans, $B M I$ body mass index

significantly. In addition, the eGFR decreased significantly during the study period, but changes in the UACR were not significant.

The changes in HbA1c $(\triangle \mathrm{HbA} 1 \mathrm{c})$ at 3 and 12 months were significantly associated with the baseline HbA1c level (Fig. 1). When the predictive factors for $\triangle \mathrm{HbA} 1 \mathrm{c}$ were examined by multiple regression analysis (see Additional file 1: Table S1), only baseline HbA1c remained a significant factor for $\triangle \mathrm{HbA} 1 \mathrm{c}$ at 3 months $(\beta=-0.608, P<0.001)$. However, at 12 months, significant determinants of $\triangle \mathrm{HbA} 1 \mathrm{c}$ were age $(\beta=-0.207$, $\mathrm{P}=0.005)$, change in body weight $(\beta=0.148$, $\mathrm{P}=0.042)$, and baseline HbA1c level $(\beta=-0.435$, $\mathrm{P}<0.001)$.

\section{Pro-inflammatory biomarkers, CV biomarkers, and CV function}

We studied pro-inflammatory biomarkers, CV biomarkers, and CV function in a subpopulation of 127 patients. The hs-CRP, BNP, and urinary 8-OHdG levels remained unchanged after 3 and 12 months of sitagliptin therapy (see Additional file 2: Table S2), but the hs-TnT level significantly increased by 12 months. When the changes in BNP, hs-TnT, and urinary $8-\mathrm{OHdG}$ were divided into groups with either a reduction or an increase, we found no association with $\triangle$ HbA1c (all $\mathrm{P}=\mathrm{n} . \mathrm{s}$.). In contrast, the changes in hs-CRP were significantly associated with changes in HbA1c at both 3 and 12 months $(P=0.030$ and 0.004 , respectively), suggesting that the effect of sitagliptin on $\mathrm{HbA1c}$ levels is blunted in patients with increased hsCRP levels (Fig. 1).

Among the ECG parameters, HR changed during the 12 months of sitagliptin therapy from $61.2 \pm 10.5$ beats per minute $(\mathrm{bpm})$ at baseline to $65.0 \pm 11.9 \mathrm{bpm}$ at 12 months $(\mathrm{P}<0.01)$. However, although the QT interval shortened significantly $(427.9 \pm 34.0 \mathrm{~ms}$ at baseline to $417.9 \pm 34.5 \mathrm{~ms}$ at 12 months, $\mathrm{P}<0.001$ ), the QTc interval did not change $(428.1 \pm 22.6 \mathrm{~ms}$ at baseline to $425.2 \pm 21.9 \mathrm{~ms}$ at 12 months, $\mathrm{P}=0.093$ ). Other parameters did not change, including the rhythm, PR interval, QRS interval, and SV1 + RV5 value. 
Table 2 Serial changes in clinical and laboratory parameters

\begin{tabular}{|c|c|c|c|c|}
\hline & Baseline & 3 months & 12 months & $P$ value \\
\hline Body weight (kg) & $67.3 \pm 14.0$ & $67.5 \pm 14.1$ & $67.9 \pm 14.1$ & 0.042 \\
\hline HbA1c (\%) & $7.09 \pm 0.81$ & $6.68 \pm 0.69$ & $6.69 \pm 0.72$ & $<0.001$ \\
\hline FPG (mg/dL) & $139.8 \pm 33.0$ & $130.2 \pm 27.2$ & $131.7 \pm 29.0$ & $<0.001$ \\
\hline $\mid \mathrm{RI}(\mathrm{IU})$ & $11.3 \pm 10.1$ & $12.4 \pm 14.3$ & $11.8 \pm 11.1$ & 0.720 \\
\hline HOMA-R & $3.9 \pm 3.8$ & $3.9 \pm 3.8$ & $3.9 \pm 3.8$ & 0.914 \\
\hline Insulin resistance (HOMA-R $\geq 2.5)(\%)$ & 54.7 & 49.4 & 51.4 & \\
\hline $\mathrm{LDL}-\mathrm{C}(\mathrm{mg} / \mathrm{dL})$ & $100.0 \pm 24.2$ & $101.4 \pm 26.8$ & $99.5 \pm 28.6$ & 0.509 \\
\hline $\mathrm{HDL}-\mathrm{C}(\mathrm{mg} / \mathrm{dL})$ & $52.4 \pm 14.1$ & $51.8 \pm 13.4$ & $50.0 \pm 12.0$ & 0.036 \\
\hline Triglyceride (mg/dL) & $136.1 \pm 77.6$ & $131.5 \pm 71.8$ & $133.6 \pm 68.4$ & 0.308 \\
\hline Uric acid (mg/dL) & $5.9 \pm 1.2$ & $6.0 \pm 1.4$ & $5.8 \pm 1.3$ & 0.054 \\
\hline $\mathrm{Cr}(\mathrm{mg} / \mathrm{dL})$ & $0.86 \pm 0.25$ & $0.88 \pm 0.27$ & $0.88 \pm 0.30$ & $<0.001$ \\
\hline $\mathrm{eGFR}\left(\mathrm{mL} / \mathrm{min} / 1.73 \mathrm{~m}^{2}\right)$ & $67.7 \pm 18.3$ & $66.3 \pm 18.3$ & $66.6 \pm 18.6$ & 0.009 \\
\hline eGFR $<60(\%)$ & 35.9 & 40.3 & 35.3 & \\
\hline UACR (mg/g Cr) & $79.8 \pm 211.4$ & $119.3 \pm 483.2$ & $100.5 \pm 239.9$ & 0.693 \\
\hline Micro- or overt-albuminuria (\%) & 40.2 & 46.3 & 39.5 & \\
\hline
\end{tabular}

Values are the mean \pm SD or $\%$

Cr Creatinine, eGFR estimated glomerular filtration rate, FPG fasting plasma glucose, $H b A 1 c$ glycated hemoglobin, $H D L-C$ high-density lipoprotein cholesterol, $H O M A-R$ homeostasis model assessment-insulin resistance, $I R I$ immunoreactive insulin, $L D L-C$ low-density lipoprotein cholesterol, $U A C R$ albumin-to-creatinine ratio

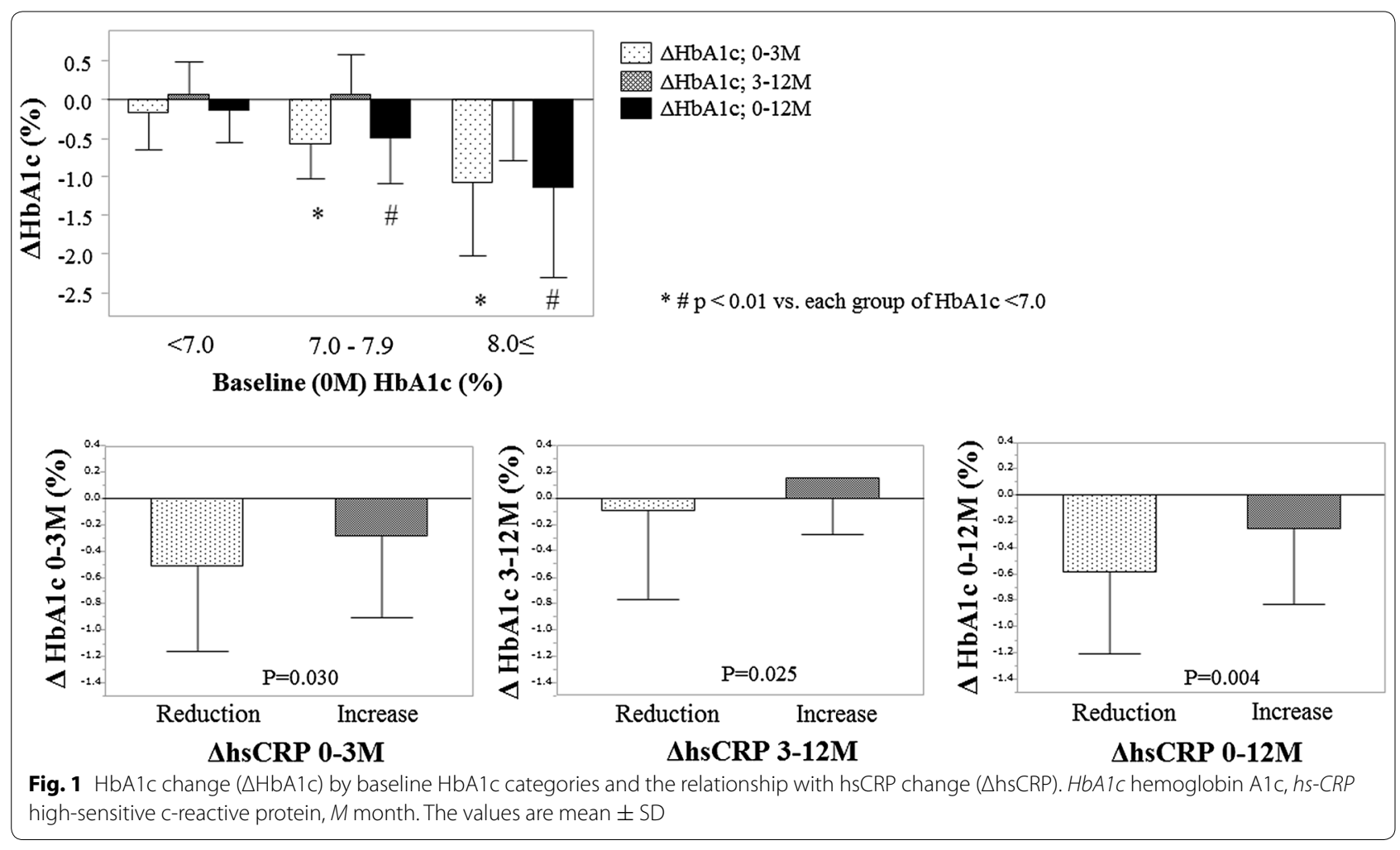

In the UCG examination, the LV mass index significantly decreased during the 12 months of treatment, from $109.9 \pm 33.5 \mathrm{~g} / \mathrm{m}^{2}$ at baseline to $105.1 \pm 31.9 \mathrm{~g} /$ $\mathrm{m}^{2}$ at 12 months $(\mathrm{P}=0.027)$. However, the other parameters, including LV dimension, EF, E/A, Dct, $\mathrm{e}^{\prime}$, and $\mathrm{E} / \mathrm{e}^{\prime}$, showed no changes between baseline and 12 months (Table 3). In the RH-PAT analysis, the RHI did not change by 3 months of sitagliptin treatment $(1.92 \pm 0.48$ 
Table 3 Changes in electrocardiography and echocardiography parameters

\begin{tabular}{|c|c|c|c|}
\hline & Baseline & 12 months & $P$ value \\
\hline \multicolumn{4}{|c|}{ Electrocardiography parameters } \\
\hline $\mathrm{HR}(\mathrm{bpm})$ & $61.2 \pm 10.5$ & $65.0 \pm 11.9$ & $<0.0001$ \\
\hline Sinus rhythm (\%) & 90.1 & 89.0 & \\
\hline PR interval (ms) & $179.5 \pm 32.2$ & $180.5 \pm 31.4$ & 0.5784 \\
\hline QRS interval (ms) & $98.0 \pm 19.1$ & $97.9 \pm 18.2$ & 0.7720 \\
\hline $\mathrm{SV} 1+\mathrm{RV} 5$ voltage $(\mathrm{mm})$ & $2.7 \pm 0.8$ & $2.6 \pm 0.7$ & 0.1288 \\
\hline QT interval (ms) & $427.9 \pm 34.0$ & $417.9 \pm 34.5$ & 0.0004 \\
\hline QTc interval (ms) & $428.1 \pm 22.6$ & $425.2 \pm 21.9$ & 0.0933 \\
\hline \multicolumn{4}{|c|}{ Echocardiography parameters } \\
\hline LV Dd (mm) & $49.9 \pm 6.3$ & $49.5 \pm 6.3$ & 0.0803 \\
\hline LV Ds (mm) & $33.2 \pm 8.2$ & $32.7 \pm 7.9$ & 0.0720 \\
\hline IVST (mm) & $8.9 \pm 1.9$ & $8.9 \pm 1.8$ & 0.7381 \\
\hline PWT (mm) & $9.3 \pm 6.0$ & $9.3 \pm 6.0$ & 0.6180 \\
\hline $\mathrm{EF}(\%)$ & $59.7 \pm 12.3$ & $60.0 \pm 11.8$ & 0.4006 \\
\hline LV mass index $\left(\mathrm{g} / \mathrm{m}^{2}\right)$ & $109.9 \pm 33.5$ & $105.1 \pm 31.9$ & 0.0270 \\
\hline E-wave velocity $(\mathrm{m} / \mathrm{s})$ & $68.2 \pm 19.5$ & $68.4 \pm 20.0$ & 0.7217 \\
\hline A-wave velocity $(\mathrm{m} / \mathrm{s})$ & $78.6 \pm 21.8$ & $80.7 \pm 20.8$ & 0.3428 \\
\hline Dct (ms) & $216.9 \pm 58.5$ & $223.9 \pm 54.8$ & 0.3279 \\
\hline E/A ratio & $0.91 \pm 0.45$ & $0.85 \pm 0.31$ & 0.2005 \\
\hline $\mathrm{e}^{\prime}(\mathrm{cm} / \mathrm{s})$ & $6.08 \pm 2.06$ & $5.87 \pm 1.59$ & 0.0938 \\
\hline E/e' ratio & $12.11 \pm 5.20$ & $12.34 \pm 5.22$ & 0.4858 \\
\hline
\end{tabular}

Values are the mean \pm SD or $\%$

Dct deceleration time, $D d$ dimension of diastole, Ds dimension of systole, EF ejection fraction, $H R$ heart rate, IVST interventricular septal thickness, $L V$ left ventricular PWT posterior wall thickness

at baseline to $1.87 \pm 0.80$ at 3 months, $P=0.648$ ), and the proportion of patients with an $\mathrm{RHI} \leq 1.67$ did not change significantly from baseline to 12 months (51.6-42.4\%).

\section{Changes in $\mathrm{BP}$ and renal function}

The results are summarized in Fig. 2. During treatment, systolic BP (SBP) and diastolic BP (DBP) were significantly reduced from baseline (SBP, $133.6 \pm 19.2 \mathrm{mmHg}$; DBP, $74.8 \pm 11.6 \mathrm{mmHg}$ ) to 12 months (SBP, $127.5 \pm 16.4 \mathrm{mmHg}$; DBP, $70.1 \pm 12.4 \mathrm{mmHg}$ ). The proportion of patients with a $\mathrm{BP} \geq 140 / 90 \mathrm{mmHg}$ significantly decreased from baseline to 12 months (33.7-25.3\%). Of the 94 patients who used an antihypertensive agent at baseline and completed the 12-month assessment period, $15(16.0 \%)$ changed the drug or the dosage during the period. When these patients were excluded, significant changes were still observed in SBP and DBP at 12 months $(-11.7 \pm 18.6$ and $-7.3 \pm 11.6 \mathrm{mmHg}$ for $\triangle \mathrm{SBP}$ and $\triangle \mathrm{DBP}$, respectively). However, $\triangle \mathrm{HbA1c}$ by 12 months was associated with neither $\triangle \mathrm{SBP}(\mathrm{r}=0.046, \mathrm{P}=0.318)$ nor $\triangle \mathrm{DBP}(\mathrm{r}=0.099, \mathrm{P}=0.199)$. In addition, HR increased from $66.9 \pm 13.0 \mathrm{bpm}$ at baseline to $71.3 \pm 12.9 \mathrm{bpm}$ at 12 months. However, $\triangle \mathrm{HR}$ was not associated with $\triangle \mathrm{SBP}$ $(\mathrm{r}=0.046, \mathrm{P}=0.563)$ or $\triangle \mathrm{DBP}(\mathrm{r}=0.036, \mathrm{P}=0.644)$ at 12 months (see Additional file 3: Figure S1).

Albuminuria at baseline was positively associated with SBP at baseline. Moreover, the changes in albuminuria $(\triangle \mathrm{AU})$ and in systolic BP $(\triangle \mathrm{SBP})$ over 12 months were significantly associated; patients with a reduction in albuminuria $(n=63)$ showed a greater reduction of SBP over the same period than those with an increase in albuminuria $(\mathrm{n}=57)(\Delta \mathrm{SBP} ;-14.3 \pm 19.3$ vs. $-6.2 \pm 14.6 \mathrm{mmHg}$, respectively). The tertile of $\triangle \mathrm{SBP}(\mathrm{n}=40$ each) was negatively correlated with a decrease in albuminuria $(\mathrm{P}=0.045)$. In contrast, although $\triangle \mathrm{SBP}$ or $\triangle \mathrm{DBP}$ was significantly associated with $\triangle \mathrm{eGFR}(\mathrm{r}=0.193, \mathrm{P}=0.012$ and $\mathrm{r}=0.256$, $\mathrm{P}<0.001$, respectively) (see Additional file 3: Figure S1), $\triangle \mathrm{AU}$ and $\triangle \mathrm{eGFR}$ were not associated after 12 months of treatment $(r=0.079, P=0.375)$. Finally, $\Delta A U$ was not associated with $\Delta \operatorname{HbA1c}(\mathrm{r}=0.046, \mathrm{P}=0.604)$.

\section{Discussion}

Gliptins can exert favorable cardiovascular effects in experimental models. These changes, as an almost general rule, include improved endothelial function, reduction of inflammatory markers, oxidative stress, ischemia/ reperfusion injury and atherogenesis. In addition, increased adiponectin levels and modest decreases in lipidemia and blood pressure were reported [8]. Unfortunately, the favorable DPP4 inhibition features reported in experimental studies are not necessarily reflected in clinical studies [7] and a definite relationship between gliptins treatment and improved $\mathrm{CV}$ outcomes remains uncertain and requires further clarification [9, 14]. This could be due to the fact that in addition to the activation of incretin hormones, gliptins are multi-target compounds and may therefore affect various additional substrates, leading to undesirable effects [15].

\section{Effect of sitagliptin on CV function and biomarkers}

Interestingly, the reduction in hs-CRP levels was independently associated with the reduction in HbA1c levels. A recent cross-over study showed that 6 weeks of treatment with sitagliptin significantly reduced the plasma levels of CRP and that there was a significant inverse correlation between changes in GLP-1 and CRP levels following sitagliptin therapy $(\mathrm{r}=0.41, \mathrm{P}=0.01)$ [16]. In the present study, sitagliptin did not significantly decrease hs-CRP levels $(P=0.0994)$, and the baseline hs-CRP level was not associated with $\triangle \mathrm{HbA1c}$ (data not shown); therefore, the causal relationship between these factors remains unknown. However, these data suggest that there is a relationship between improvements in glucose-insulin homeostasis and low-grade inflammation that is somehow mediated by sitagliptin therapy in diabetic subjects with high $\mathrm{CV}$ risk. 


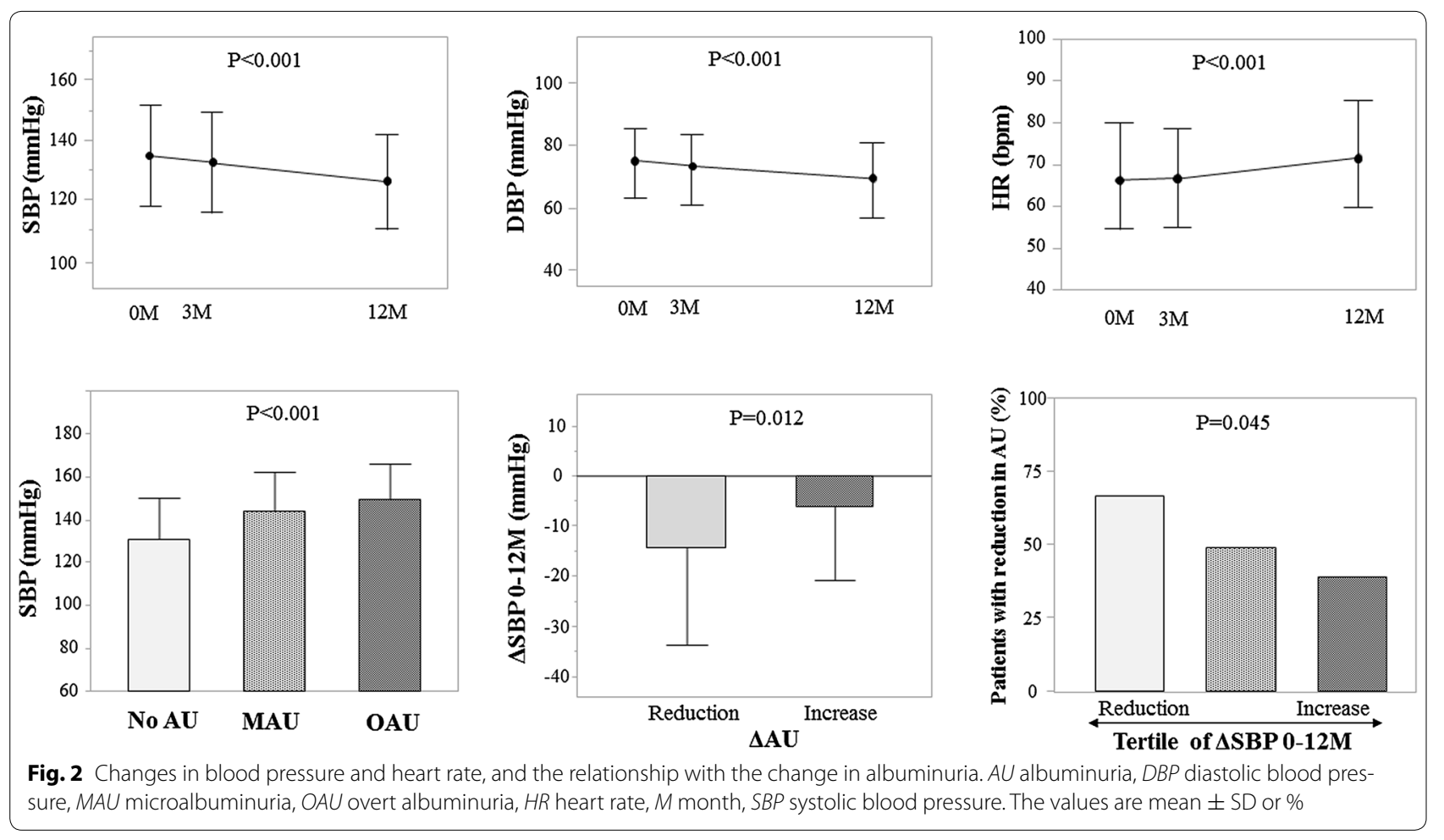

Prolongation of the QT interval is an important risk factor for ventricular arrhythmia and sudden death. In the present study, the QT interval was significantly shortened from baseline to 12 months $(\mathrm{P}<0.001)$, but there was no significant difference in the QT interval after correction for the change in HR (QTc interval) $(\mathrm{P}=0.093)$. The basic research by Lee et al. showed that sitagliptin treatment could reverse calcium regulation in cardiomyocytes, which might contribute to its shortening effects on the QT interval in hypertensive animals [17]. In other research, sitagliptin was not associated with prolongation of either the QT or QTc interval at clinically relevant concentrations in healthy individuals [18]. Although the shortening of the QT interval in this study was mainly associated with the increased HR, the QTc interval still showed a trend toward shortening after sitagliptin therapy $(\mathrm{P}=0.093)$. Thus, it is possible that sitagliptin therapy might lower the risks of ventricular arrhythmias and sudden death due to QT interval change, but further studies are clearly necessary to clarify whether benefits truly exist, particularly in patients with diabetes and comorbid CVD.

GLP-1 receptors are located in cardiac myocytes, coronary vascular endothelial cells, and smooth muscle cells; and, mainly in basic studies, GLP-1 has been reported to improve cardiac function and provide cardioprotective effects [9]. Nikolaidis et al. reported that GLP-1 infusion in patients with acute myocardial infarction improved LV function compared with controls receiving standard care [19]. However, in a randomized study of 80 diabetic patients with LV diastolic dysfunction, Oe et al. reported that sitagliptin for 6 months did not improve diastolic function [20]. In the present study, in which we used patients with similar background characteristics, there was no significant change in BNP levels at 12 months, which is a reasonably sensitive maker for the cardiac dysfunction and development of HF. This was the same for the EF and septal $\mathrm{e}^{\prime}$ or $E / \mathrm{e}^{\prime}$, which reflect diastolic function or end-diastolic pressure, as the diagnostic and therapeutic index of HF. Endothelial dysfunction may also result from hyperglycemia and atherosclerosis, and some studies have suggested that sitagliptin can improve endothelial dysfunction in patients with type 2 diabetes and that gliptins may protect endothelial function through a GLP-1-dependent mechanism [21, 22]. In contrast, Aoyagi et al. recently reported that gliptins attenuated endothelial function evaluated by flow-mediated vasodilatation [23]. Also, sitagliptin has been shown to increase adiponectin levels [24, 25], which could be associated with the anti-atherosclerotic effects. Although the measurement was not performed in the present study, sitagliptin treatment did not reduce markers of oxidative stress (urinary 8-OHdG) or inflammation (hs-CRP) but decreased HDL cholesterol levels. They did not affect endothelial function (RHI assessment) favorably. Thus, controversy remains around these aspects of treatment, and further investigation is needed. 


\section{Effect of sitagliptin on BP and albuminuria}

We showed that sitagliptin reduced SBP and DBP independent of $\triangle \mathrm{HbA} 1 \mathrm{c}$ and that this was associated with a reduction in the level of albuminuria. It might also have been associated with a reduction in the LV mass index. Relatively few studies have examined the effects of DPP-4 inhibitors on BP in patients with diabetes [26]. Presently, the effects are unclear and controversial in both clinical and basic studies, with some investigators reporting that gliptins may reduce BP [27-29] and others reporting that these agents have no significant effects on BP [30,31]. Ogawa et al. reported sitagliptin treatment affected SBP but not DBP in 17 patients with hypertension; however, in that research, the degree of decrease in HbA1c levels did not significantly correlate with the degree of decrease in SBP $(r=0.24)$ [28]. The results were, therefore, concordant with the present result in patients with mild diabetes but at high $\mathrm{CV}$ risk.

DPP-4 inhibition with sitagliptin increases the activity of GLP-1, which has been reported to decrease urinary salt uptake and increase urinary salt excretion [32]. In fact, 6 months of treatment with exenatide, a GLP-1 receptor agonist, was associated with a greater reduction in SBP compared with placebo or insulin in pooled data from six trials including 2171 patients [33]. The DURATION-2 study assessed the safety and efficacy of once weekly exenatide against maximum approved doses of sitagliptin, thiazolidinedione, or pioglitazone, and indicated that the reduction in SBP was significantly greater with exenatide once weekly than with sitagliptin in all patients after 26 weeks of treatment [34]. The reason for the variation in the long-term effects of gliptins on BP remains unknown; however, it might be dependent on the patient's physiological state because DPP-4 metabolizes both prohypertensive and antihypertensive peptides [35]. Further clinical studies are necessary to elucidate the reasons for these differences. In the present study, HR significantly increased both in ECG analysis and ambulatory $\mathrm{BP}$ measurement. It might be associated with the reactive activation in the sympathetic nerve system or inhibition of the vagal nerve system to the BP decrease as suggested in the treatment of GLP-1 receptor agonists [36]. However, the $\triangle \mathrm{HR}$ was not associated with $\triangle \mathrm{SBP}$ or $\triangle \mathrm{DBP}$ at 12 months in the present study, and the other mechanism might be suggested. The point of the long-term effects or CV outcomes needs to be clarified with caution [37].

The development of albuminuria is a key step in the progression of diabetic kidney disease, and worsening of albuminuria is a significant predictor of both progressive renal disease and CVD in patients with diabetes. The mechanisms underlying albuminuria are complex, and improved insulin sensitivity, weight loss, reduced blood glucose levels, lower BP, and suppressed inflammation might all contribute to a reduction in albuminuria. Several studies have reported a significant correlation between improvements of albuminuria and BP in patients with diabetes, hypertension, or nephropathy. Kawasaki et al. reported in a retrospective analysis that sitagliptin reduced albuminuria, and that this was independently associated with reductions in BP and eGFR in 247 patients with diabetes [38]. In the present study, although sitagliptin did not reduce albuminuria overall, the reduction of BP was modestly associated with a decrease in albuminuria that was independent of $\Delta \mathrm{HbA} 1 \mathrm{c}$. Groop et al. reported that treatment with linagliptin for diabetic patients with renal dysfunction led to a significant reduction in albuminuria, independent of changes in SBP [39]. Although these findings may suggest direct renoprotective effects of DPP-4 inhibitors [40], such a blood-pressure-independent effect was not observed in the present study. eGFR was deteriorated during the 12-month sitagliptin treatment, which was positively associated with $\triangle \mathrm{SBP}$ or $\triangle \mathrm{DBP}$ in the present study. Kawasaki et al. reported the similar findings and discussed that it might be related to the decrease in the glomerular internal pressure and be beneficial for the renal tissue [38]. This mechanism resembles the decrease in eGFR observed through the action of renin-angiotensin system inhibitors in decreasing glomerular internal pressure and albuminuria. Further evidence is needed to confirm these renal effects, particularly in the long-term and explore the underlying mechanisms in patients with diabetes [41].

\section{Study limitations}

First, this was an uncontrolled study and the effects of sitagliptin were only assessed by comparison of measurements at three independent time points. It might be possible that the significant changes in several measures were affected by factors other than sitagliptin treatment. However, all the patients were educated about the importance of diet and physical exercise, and most had stable medical treatment at least 1 month before the study inclusion. Second, the study assessments took place at 3 or 12 months, and may have been too short for the assessment of $\mathrm{CV}$ effects and outcomes. Therefore, the analysis may have been insufficient to provide conclusive evidence for long-term CV effects and outcomes associated with sitagliptin. Future studies must examine the BP lowering effects of sitagliptin and its direct influence on CV outcomes. Third, most of the participants had earlystage diabetes $(69.3 \%$ had an HbA1c $<7.0 \%)$ and higher age (73.4 \% were aged $>65$ years), meaning that our results cannot be extrapolated to all patients with diabetes and high CV risk. Finally, our study design also led to the exclusion of some patients with severe chronic kidney disease or end-stage renal disease, which limits the applicability of the data in these settings. 


\section{Conclusion}

We conducted a prospective, multicenter, observational study of 205 patients with type 2 diabetes at high risk of CVD who were treated with sitagliptin for 12 months. The efficacy of sitagliptin for glycemic control was demonstrated by the higher baseline HbA1c level and the reduction of hs-CRP levels. Sitagliptin also showed BP lowering effects over the 12-month study period associated with the prevention of microalbuminuria progression. However, there were no beneficial effects on cardiac and endothelial function or on serum BNP, hs-TnT, and urinary 8-OHdG levels. We conclude that in Japanese patients with diabetes at high risk of CVD, sitagliptin had beneficial effects on BP and glycemic control over 12 months and it might offer long-term advantages in the management of diabetes with comorbid CVD.

\section{Additional files} Additional file 1: Table S1. Comparison of changes in $\mathrm{HbA} 1 \mathrm{c}(\triangle \mathrm{HbA} 1 \mathrm{c})$
at different time periods by multivariate prediction models.

Additional file 2: Table S2. Changes in pro-inflammatory and cardiovascular biomarkers.

Additional file 3: Figure S1. Relationship of changes in blood pressure ( $\triangle \mathrm{SBP}$ or $\triangle \mathrm{DBP}$ ) with those in heart rate $(\triangle \mathrm{HR}$ ) or those in eGFR ( $\triangle \mathrm{eGFR})$ for 12 months.

\begin{abstract}
Abbreviations
AU: albuminuria; BNP: B-type natriuretic peptide; BP: blood pressure; CV: cardiovascular; CVD: cardiovascular disease; Dct: deceleration time of the mitral E-wave velocity; DPP-4: dipeptidyl peptidase-4; e': peak of myocardial early diastolic velocity; E/A ratio: ratio of peak mitral E-wave velocity to peak mitral A-wave velocity; ECG: electrocardiography; EF: ejection fraction; eGFR: estimated glomerular filtration rate; GIP: glucose-dependent insulinotropic polypeptide; GLP-1: glucagon-like peptide-1; HbA1c: hemoglobin A1c; HDL: high-density lipoprotein; HOMA-IR: homeostasis model assessmentinsulin resistance; HR: heart rate; hs-CRP: high-sensitive C-reactive protein; hs-TnT: high-sensitive troponin T; LV: left ventricular; 8-OHdG: 8-hydroxy-2'deoxyguanosine; $\mathrm{RHI}$ : reactive hyperemia index; $\mathrm{RH}-\mathrm{PAT}$ : reactive hyperemiaperipheral arterial tonometry; SD: standard deviation; UACR: urinary albuminto-creatinine ratio; UCG: echocardiography.
\end{abstract}

\section{Authors' contributions \\ The contribution of each author to this study was as follows: Takashi Naka- mura and Yoshitaka Iwanaga, the idea and design of the study, analysis of the data, and writing of the manuscript; Yuki Miyaji, analysis of the data; and Ryuji Nohara, Takao Ishimura, and Shunichi Miyazaki, the idea and design of the study, interpretation of data, and writing of the manuscript. All authors read and approved the final manuscript.}

\section{Author details}

${ }^{1}$ Division of Cardiology, Department of Internal Medicine, Faculty of Medicine, Kinki University, 377-2 Ohno-Higashi, Osakasayama 589-8511, Japan. ${ }^{2}$ Hirakata Kousai Hospital, Hirakata, Japan. ${ }^{3}$ Ishimura Clinic, Osaka, Japan.

\section{Acknowledgements}

This study was supported by the Osaka Foundation for the Prevention of Cancer and Cardiovascular Disease and we acknowledge their sponsorship. We also would like to acknowledge Ms. Hisako Fujimoto and Asako Hasegawa (Nouvelle Place, Inc.) for their excellent secretarial assistance. SIRKAS Investigators: Department of Internal Medicine, Kinki University Faculty of Medicine, Osakasayama, Japan (Yuki Miyaji, Takashi Nakamura, Yoshitaka
Iwanaga, Shunichi Miyazaki); Ishimura Clinic, Osaka (Takao Ishimura); The Tazuke Kofukai Medical Research Institute, Kitano Hospital, Osaka, (Toshiaki Izumi, Moriaki Inoko, Ryuji Nohara), Department of Cardiology, Kishiwada City Hospital, Kishiwada (Mitsuo Matsuda); Department of Cardiovascular Center, Osaka Red Cross Hospital, Osaka (Tsukasa Inada, Masaru Tanaka); Department of Cardiology, Tenri Hospital, Tenri (Hirokazu Kondo, Yoshihisa Nakagawa); Department of Cardiovascular Medicine, Osaka City University Graduate School of Medicine, Osaka (Akihisa Hanatani, Minoru Yoshiyama); Nissei Hospital, Osaka (Atsushi Nakagawa); Takanohara Central Hospital, Nara (Taku Hirai), Department of Metabolic Medicine, Osaka University Graduate School of Medicine, Osaka (Akihisa Imagawa).

\section{Competing interests}

Shunichi Miyazaki received the research funding from the following companies; MSD, Daiichi-Sankyo, Otsuka, Boehringer Ingelheim, Astellas Pharma companies. Ryuji Nohara received the research funding from the following companies: Astellas Pharma, Toa Eiyo, Boehringer Ingelheim, Daiichi-Sankyo, and Astra Zeneca.

Received: 25 February 2016 Accepted: 18 March 2016

Published online: 31 March 2016

\section{References}

1. Mak KH, Moliterno DJ, Granger CB, Miller DP, White HD, Wilcox RG, Califf RM, Topol EJ. Influence of diabetes mellitus on clinical outcome in the thrombolytic era of acute myocardial infarction. GUSTO-I investigators. Global utilization of streptokinase and tissue plasminogen activator for occluded coronary arteries. J Am Coll Cardiol. 1997;30:171-9.

2. Mazzone T, Chait A, Plutzky J. Cardiovascular disease risk in type 2 diabetes mellitus: insights from mechanistic studies. Lancet. 2008;371:1800-9.

3. Tabas I. The role of endoplasmic reticulum stress in the progression of atherosclerosis. Circ Res. 2010;107:839-50.

4. Beckman JA, Creager MA, Libby P. Diabetes and atherosclerosis: epidemiology, pathophysiology, and management. JAMA. 2002;287:2570-81.

5. Gaede $\mathrm{P}$, Lund-Andersen $\mathrm{H}$, Parving HH, Pedersen O. Effect of a multifactorial intervention on mortality in type 2 diabetes. N Engl J Med. 2008;358:580-91.

6. Baggio LL, Drucker DJ. Biology of incretins: GLP-1 and GIP. Gastroenterology. 2007;132:2131-57.

7. Green JB, Bethel MA, Armstrong PW, Buse JB, Engel SS, Garg J, Josse R, Kaufman KD, Koglin J, Korn S, Lachin JM, McGuire DK, Pencina MJ, Standl E, Stein PP, Suryawanshi S, de Van Werf F, Peterson ED, Holman RR, TECOS Study Group. Effect of sitagliptin on cardiovascular outcomes in type 2 diabetes. N Engl J Med. 2015;373:232-42.

8. Son JW, Kim S. Dipeptidyl peptidase 4 inhibitors and the risk of cardiovascular disease in patients with type 2 diabetes: a tale of three studies. Diabetes Metab J. 2015;39:373-83.

9. Ussher JR, Drucker DJ. Cardiovascular biology of the incretin system. Endocr Rev. 2012;33:187-215.

10. Matsuo S, Imai E, Horio M, Yasuda Y, Tomita K, Nitta K, Yamagata K, Tomino Y, Yokoyama H, Hishida A. Revised equations for estimated GFR from serum creatinine in Japan. Am J Kidney Dis. 2009;53:982-92.

11. Sano M, Aizawa Y, Katsumata Y, Nishiyama N, Takatsuki S, Kamitsuji S, Kamatani N, Fukuda K. Evaluation of differences in automated QT/QTC measurements between Fukuda Denshi and Nihon Koden systems. PLoS One. 2014;9:e106947.

12. Lang RM, Bierig M, Devereux RB, Flachskampf FA, Foster E, Pellikka PA, Picard MH, Roman MJ, Seward J, Shanewise J, Solomon S, Spencer KT, St John Sutton M, Stewart W, American Society of Echocardiography's Nomenclature and Standards Committee, Task Force on Chamber Quantification, American College of Cardiology Echocardiography Committee, American Heart Association, European Association of Echocardiography, European Society of Cardiology. Recommendations for chamber quantification. Eur J Echocardiogr. 2006;7:79-108.

13. Hamburg NM, Keyes MJ, Larson MG, Vasan RS, Schnabel R, Pryde MM, Mitchell GF, Sheffy J, Vita JA, Benjamin EJ. Cross-sectional relations of 
digital vascular function to cardiovascular risk factors in the Framingham Heart Study. Circulation. 2008;117:2467-74

14. Fisman EZ, Tenenbaum A. Antidiabetic treatment with gliptins: focus on cardiovascular effects and outcomes. Cardiovasc Diabetol. 2015;14:129.

15. Costante R, Stefanucci A, Carradori S, Novellino E, Mollica A. DPP-4 inhibitors: a patent review (2012-2014). Expert Opin Ther Pat. 2015:25:209-36.

16. Tremblay AJ, Lamarche B, Deacon CF, Weisnagel SJ, Couture P. Effects of sitagliptin therapy on markers of low-grade inflammation and cell adhesion molecules in patients with type 2 diabetes. Metabolism. 2014;63:1141-8.

17. Lee TI, Kao YH, Chen YC, Huang JH, Hsu MI, Chen YJ. The dipeptidyl peptidase-4 inhibitor-sitagliptin modulates calcium dysregulation, inflammation, and PPARs in hypertensive cardiomyocytes. Int J Cardiol. 2013;168:5390-5.

18. Bloomfield DM, Krishna R, Hreniuk D, Hickey L, Ghosh K, Bergman AJ, Miller J, Gutierrez MJ, Stoltz R, Gottesdiener KM, Hermann GA, Wagner JA. A through QTc study to assess the effect of sitagliptin, a DPP4 inhibitor, on ventricular repolarization in healthy subjects. J Clin Pharmacol. 2009;49:937-46.

19. Nikolaidis LA, Mankad S, Sokos GG, Miske G, Shah A, Elahi D, Shannon RP. Effects of glucagon-like peptide- 1 in patients with acute myocardial infarction and left ventricular dysfunction after successful reperfusion. Circulation. 2004;109:962-5.

20. Oe H, Nakamura K, Kihara H, Shimada K, Fukuda S, Takagi T, Miyoshi T, Hirata K, Yoshikawa J. Ito H; FESC, for Effect of a DPP-4 inhibitor on left ventricular diastolic dysfunction in patients with type 2 diabetes and diabetic cardiomyopathy (3D) study investigators. Comparison of effects of sitagliptin and voglibose on left ventricular diastolic dysfunction in patients with type 2 diabetes: results of the 3D trial. Cardiovasc Diabetol. 2015;14:83.

21. Matsubara J, Sugiyama S, Akiyama E, Iwashita S, Kurokawa H, Ohba K, Maeda H, Fujisue K, Yamamoto E, Kaikita K, Hokimoto S, Jinnouchi H, Ogawa H. Dipeptidyl peptidase-4 inhibitor, sitagliptin, improves endothelial dysfunction in association with its anti-inflammatory effects in patients with coronary artery disease and uncontrolled diabetes. Circ J. 2013;77:1337-44.

22. Nakamura K, Oe H, Kihara H, Shimada K, Fukuda S, Watanabe K, Takagi T, Yunoki K, Miyoshi T, Hirata K, Yoshikawa J, Ito H. DPP-4 inhibitor and alpha-glucosidase inhibitor equally improve endothelial function in patients with type 2 diabetes: EDGE study. Cardiovasc Diabetol. 2014;13:110.

23. Ayaori M, Iwakami N, Uto-Kondo H, Sato H, Sasaki M, Komatsu T, lizuka M, Takiguchi S, Yakushiji E, Nakaya K, Yogo M, Ogura M, Takase B, Murakami T, Ikewaki K. Dipeptidyl peptidase-4 inhibitors attenuate endothelial function as evaluated by flow-mediated vasodilatation in type 2 diabetic patients. J Am Heart Assoc. 2013;2:e003277.

24. Hibuse T, Maeda N, Kishida K, Kimura T, Minami T, Takeshita E, Hirata A, Nakagawa Y, Kashine S, Oka A, Hayashi M, Nishizawa H, Funahashi T, Shimomura I. A pilot three-month sitagliptin treatment increases serum adiponectin level in Japanese patients with type 2 diabetes mellitus- a randomized controlled trial START-J study. Cardiovasc Diabetol. 2014;13:96.

25. Omoto S, Taniura T, Nishizawa T, Tamaki T, Shouzu A, Nomura S. Antiatherosclerotic effects of sitagliptin in patients with type 2 diabetes mellitus. Diabetes Metab Syndr Obes. 2015;27(8):339-45.

26. Petrie JR. The cardiovascular safety of incretin-based therapies: a review of the evidence. Cardiovasc Diabetol. 2013;12:130.
27. Mistry GC, Maes AL, Lasseter KC, Davies MJ, Gottesdiener KM, Wagner JA Herman GA. Effect of sitagliptin, a dipeptidyl peptidase-4 inhibitor, on blood pressure in nondiabetic patients with mild to moderate hypertension. J Clin Pharmacol. 2008;48:592-8.

28. Ogawa S, Ishiki M, Nako K, Okamura M, Senda M, Mori T, Ito S. Sitagliptin, a dipeptidyl peptidase-4 inhibitor, decreases systolic blood pressure in Japanese hypertensive patients with type 2 diabetes. Tohoku J Exp Med. 2011;223:133-5.

29. Pacheco BP, Crajoinas RO, Couto GK, Davel AP, Lessa LM, Rossoni LV, Girardi AC. Dipeptidyl peptidase IV inhibition attenuates blood pressure rising in young spontaneously hypertensive rats. J Hypertens. 2011;29:520-8.

30. von Eynatten M, Gong Y, Emser A, Woerle HJ. Efficacy and safety of linagliptin in type 2 diabetes subjects at high risk for renal and cardiovascular disease: a pooled analysis of six phase III clinical trials. Cardiovasc Diabetol. $2013 ; 12: 60$.

31. Chaykovska L, Alter ML, von Websky K, Hohmann M, Tsuprykov O, Reichetzeder C, Kutil B, Kraft R, Klein T, Hocher B. Effects of telmisartan and linagliptin when used in combination on blood pressure and oxidative stress in rats with 2-kidney-1-clip hypertension. J Hypertens. 2013;31:2290-8.

32. Gutzwiller JP, Hruz P, Huber AR, Hamel C, Zehnder C, Drewe J, Gutmann $H$, Stanga Z, Vogel D, Beglinger C. Glucagon-like peptide- 1 is involved in sodium and water homeostasis in humans. Digestion. 2006;73:142-50.

33. Okerson T, Yan P, Stonehouse A, Brodows R. Effects of exenatide on systolic blood pressure in subjects with type 2 diabetes. Am J Hypertens. 2010;23:334-9.

34. Bergenstal RM, Wysham C, Macconell L, Malloy J, Walsh B, Yan P, Wilhelm K, Malone J, Porter LE. DURATION-2 Study Group. Efficacy and safety of exenatide once weekly versus sitagliptin or pioglitazone as an adjunct to metformin for treatment of type 2 diabetes (DURATION-2): a randomised trial. Lancet. 2010;376:431-9.

35. Jackson EK, Mi Z, Tofovic SP, Gillespie DG. Effect of dipeptidyl peptidase 4 inhibition on arterial blood pressure is context dependent. Hypertension. 2015;65:238-49.

36. Valensi P, Chiheb S, Fysekidis M. Insulin- and glucagon-like peptide1-induced changes in heart rate and vagosympathetic activity: why they matter. Diabetologia. 2013;56:1196-200.

37. Carnethon MR, Yan L, Greenland P, Garside DB, Dyer AR, Metzger B, Daviglus ML. Resting heart rate in middle age and diabetes development in older age. Diabetes Care. 2008;31:335-9.

38. Kawasaki I, Hiura Y, Tamai A, Yoshida Y, Yakusiji Y, Ikuno Y, Okada M, Ueno H, Tanaka N, Yamagami K, Fukumoto M, Hosoi M. Sitagliptin reduces the urine albumin-to-creatinine ratio in type 2 diabetes through decreasing both blood pressure and estimated glomerular filtration rate. J Diabetes. 2015;7:41-6.

39. Groop PH, Cooper ME, Perkovic V, Emser A, Woerle HJ, von Eynatten M. Linagliptin lowers albuminuria on top of recommended standard treatment in patients with type 2 diabetes and renal dysfunction. Diabetes Care. 2013;36:3460-8.

40. Fujita H, Taniai H, Murayama H, Ohshiro H, Hayashi H, Sato S, Kikuchi N, Komatsu T, Komatsu K, Komatsu K, Narita T, Yamada Y. DPP-4 inhibition with alogliptin on top of angiotensin II type 1 receptor blockade ameliorates alubuminuria via up-regulation of SDF-1a in type 2 diabetic patients with incipient nephropathy. Endocr J. 2014;61:159-66.

41. Avogaro A, Fadini GP. The effects of dipeptidyl peptidase-4 inhibition on microvascular diabetes complications. Diabetes Care. 2014;37:2884-94. 